Revista Arbitrada Interdisciplinaria KOINONIA

Año VI. Vol VI. N¹1. Enero - Junio. 2021

Hecho el depósito de Ley: FA2016000010

ISSN: $2542-3088$

FUNDACIÓN KOINONIA (F.K). Santa Ana de Coro. Venezuela.

Axel Alejandro Macías-Rodríguez; Luís Javier Molina-Chalacán; Edmundo Jalón-Arias

http://dx.doi.org/10.35381/r.k.v6i11.1197

\title{
Aplicativo web para la búsqueda y ubicación de libros en bibliotecas de Instituciones Escolares
}

Web application for the search and location of books in libraries of School Institutions

Axel Alejandro Macías-Rodríguez

sq.axelamr95@uniandes.edu.ec

Universidad Regional Autónoma de los Andes, Quevedo

Ecuador

https://orcid.org/0000-0002-7452-7687

Luís Javier Molina-Chalacán

uq.luismolina@uniandes.edu.ec

Universidad Regional Autónoma de los Andes, Quevedo

Ecuador

https://orcid.org/0000-0003-3755-2717

Edmundo Jalón-Arias

uq.edmundojalon@uniandes.edu.ec

Universidad Regional Autónoma de los Andes, Quevedo

Ecuador

https://orcid.org/0000-0002-3060-736X

Recepción: 20 de diciembre 2020

Revisado: 15 de enero 2021

Aprobación: 20 de febrero 2021

Publicación: 01 de marzo 2021 


\title{
RESUMEN
}

La investigación tiene como objetivo principal implementar un aplicativo web que permita mejorar la búsqueda, ubicación, de los libros de forma sistematizada, organizada, en instituciones públicas y privadas del Ecuador. Se generó desde una concepción metodológica descriptiva documental con diseño no experimental, generando un aplicativo web, el método utilizado para el aplicativo web de búsqueda y ubicación de libros en biblioteca es la metodología RAD (Desarrollo Rápido de Aplicaciones). Se desarrolló la aplicación web para la búsqueda y ubicación de libros donde se evidenció que posee una interfaz más amigable y llamativa, un flujo de código y estructura más eficiente, y reportes con mayor comprensión. Las pruebas implementadas de implementar el aplicativo web, indican que su funcionamiento es eficiente y adecuado, además permite una óptima búsqueda de los libros que el usuario así lo requiera o necesite, de tal forma que ayuda a ahorrar el factor tiempo.

Descriptores: Automatización de bibliotecas; aplicación informática; informatización de archivos. (Palabras tomadas del Tesauro UNESCO).

\begin{abstract}
The main objective of the research is to implement a web application that allows improving the search, location, of books in a systematic, organized way, in public and private institutions in Ecuador. It was generated from a documentary descriptive methodological conception with a non-experimental design, generating a web application, the method used for the web application to search and locate books in the library is the RAD methodology (Rapid Development of Applications). The web application was developed for the search and location of books where it was evidenced that it has a more friendly and attractive interface, a more efficient code flow and structure, and reports with greater understanding. The tests implemented to implement the web application indicate that its operation is efficient and adequate, in addition it allows an optimal search of the books that the user requires or needs, in such a way that it helps to save the time factor.
\end{abstract}

Descriptors: Library automation; computer applications; archive automation. (Words taken from the UNESCO Thesaurus). 


\section{INTRODUCCIÓN}

Es evidente que en los últimos años la tecnología ha incrementado de una manera extraordinaria, de modo que ha causado un gran impacto en diferentes sectores, además, ha formado parte fundamental dentro del ámbito educativo, a tal punto que se han desarrollado plataformas virtuales que son capaces de buscar o indagar información dependiendo de las necesidades del usuario, incorporando así modelos de investigación para lograr formar individuos con espíritu investigador.

En el Ecuador la utilización de esta herramienta está avanzando continuamente, en función de que diversas instituciones tanto públicas como privadas, cuentan con bibliotecas virtuales donde se almacena información importante como artículos, papers, tesis, proyectos investigativos, revistas científicas, los cuales la mayoría se encuentran registrados digitalmente con su respectivo código, titulo, autor, editorial, año de publicación, idioma, y observación, de modo que el usuario o estudiante mediante la red puede acceder a documentos desde cualquier lugar en busca de información (QuezadaSarmiento, 2017).

Sin embargo, se ha observado que el problema se encuentra en que en algunas instituciones respecto al diseño web y a la búsqueda que presentan las bibliotecas virtuales o digitales no es la adecuada, por lo que en ciertas ocasiones provoca confusión y desconcierto al usuario o estudiante, que al momento de averiguar la información que se necesite no se encuentre como tiene que ser visualizado, por cuanto los resultados de búsqueda se muestran de una forma desordenada o poco entendible (HernándezPacheco, 2013).

De acuerdo a lo que indica (Sanz, 2016), para realizar contenido en un sitio web se debe tener en cuenta dos factores fundamentales: el primero se refiere al diseño en general y el segundo es el diseño web en particular, debido a que son disciplinas complejas y no exentas de múltiples interpretaciones, de igual manera el diseño aumenta con el tiempo y es sensible a prácticas o a tendencias visuales (Meneses-Tello, 2013). 
Por otra parte (Cruz, 2011), indica que las actividades principales en una biblioteca virtual son diseminar, generar, participar, difundir e interactuar con los usuarios o estudiantes, además, deben ser medidas por las herramientas disponibles en la actualidad como es las que se hallan en la Web (Guerrero, et al., 2015). Es importante mencionar que las características de estas tecnologías se dan de forma gratuita y las instituciones educativas se amparan a la tendencia de utilizar software libre por su rápida eficiencia y desarrollo de sitios web, por ello, una vez que se implemente la aplicación web se podrá determinar si la gestión de préstamo de material bibliográfico se realiza de manera más ágil de la que se lo hacía anteriormente (Chiroles-Cantera, et al., 2017).

En resumen, la investigación tiene como alcance mejorar el proceso de búsqueda y ubicación de los libros en las bibliotecas para las instituciones escolares, de modo que permita reducir el tiempo que se necesite para la obtención de los libros que el usuario requiera, para lo cual y en función a lo descrito con anterioridad se tiene como objetivo principal implementar un aplicativo web que permita mejorar la búsqueda, ubicación, de los libros de forma sistematizada, organizada, en instituciones públicas y privadas del Ecuador.

\section{MÉTODO}

La investigación se generó desde una concepción metodológica descriptiva documental con diseño no experimental (Aldana-Zavala, 2017), generando un aplicativo web. Para el diseño de la biblioteca digital se usaron técnicas bibliotecológicas de organización de la información teniendo en cuenta los diversos aspectos que favorecen la interacción del usuario con la página. El diseño y elaboración de la biblioteca virtual, concuerda con la estructura elaborada, posee una interfaz amigable y entendible, el cual permite realizar búsqueda de forma eficiente y segura. 


\section{Procedimiento}

En este sentido, el método utilizado para el aplicativo web de búsqueda y ubicación de libros en biblioteca es la metodología RAD (Desarrollo Rápido de Aplicaciones), (Carrizo, \& Alfaro, 2018), el cual está constituido en diversas fases que se describen a continuación:

FASE I. Etapa de investigación: En esta etapa se recopilo la información necesaria acerca de las bibliotecas virtuales y la importancia que tiene dentro de las Instituciones Educativas.

FASE II. Planeación: Dentro de ella se muestra un panorama general del aplicativo web a realizarse. Además, se tiene en cuenta todos los requerimientos funcionales y no funcionales que debe poseer el Proyecto.

FASE III. Diseño y elaboración: Se trabaja en los esquemas y prototipos de las páginas web de los módulos del sistema y ejecuta los casos de uso de cada módulo que tendrá el aplicativo web, teniendo en cuenta cada rol de usuario que la aplicación posee. Los lenguajes de programación que se utilizaron en esta parte fueron Php, Html, SQL y Css. FASE IV. Construcción: Durante esta fase se efectuará toda la construcción de la aplicación web, por lo que dentro de ella se contiene el esquema de base de datos, la codificación importante de cada módulo y las validaciones.

FASE V. Fase de transición: En esta fase se realizarán todas las pruebas correspondientes y necesarias de la aplicación web, según el módulo correspondiente. También se deberá hacer la entrega formal de la aplicación web con las instrucciones necesarias de la misma.

\section{RESULTADOS}

El conocimiento bibliográfico más las nuevas tecnologías permiten alcanzar un manejo óptimo de la información encaminada a los usuarios referente a sus necesidades internas, como el fortalecimiento en las acciones académicas, intelectuales y personales. Gracias a los diferentes avances científicos y tecnológicos que se experimentan, las 
bibliotecas virtuales surgen como intermediarias entre el usuario y el medio externo, gestionando la información para ofrecer a los usuarios la búsqueda eficiente cubriendo así sus expectativas de información (Silva-Blancas \& Romero-González, 2018).

Como resultado de esta investigación se propone el empleo de un aplicativo web para la búsqueda y ubicación de libros en bibliotecas de instituciones escolares, con el fin de que proporcione y garantice el acceso rápido y pertinente a una gran variedad de información a todos los usuarios, especialmente a los estudiantes, investigadores y docentes.

Dicha propuesta mencionada anteriormente surge para mejorar la problemática de acceso a la información que afecta a los usuarios y/o estudiantes. Por lo tanto, se ofrece una herramienta tecnológica como un método de ayuda, por medio de la cual los usuarios y/o estudiantes podrán investigar y navegar sin algún tipo de limitaciones o barreras y puedan obtener toda la información que así lo requieran. La biblioteca virtual se asume como un producto y una herramienta tecnológica que tendrá el propósito de mostrar y divulgar sus materiales bibliográficos. 


\section{PRESENTACIÓN DE LA BIBLIOTECA VIRTUAL}

La página que se mostrará a continuación simboliza el esquema o los contenidos de cada parte de la biblioteca virtual. Dentro de ella se presenta las diferentes opciones importantes tales como ingreso, creación de cuenta, categoría, contacto.

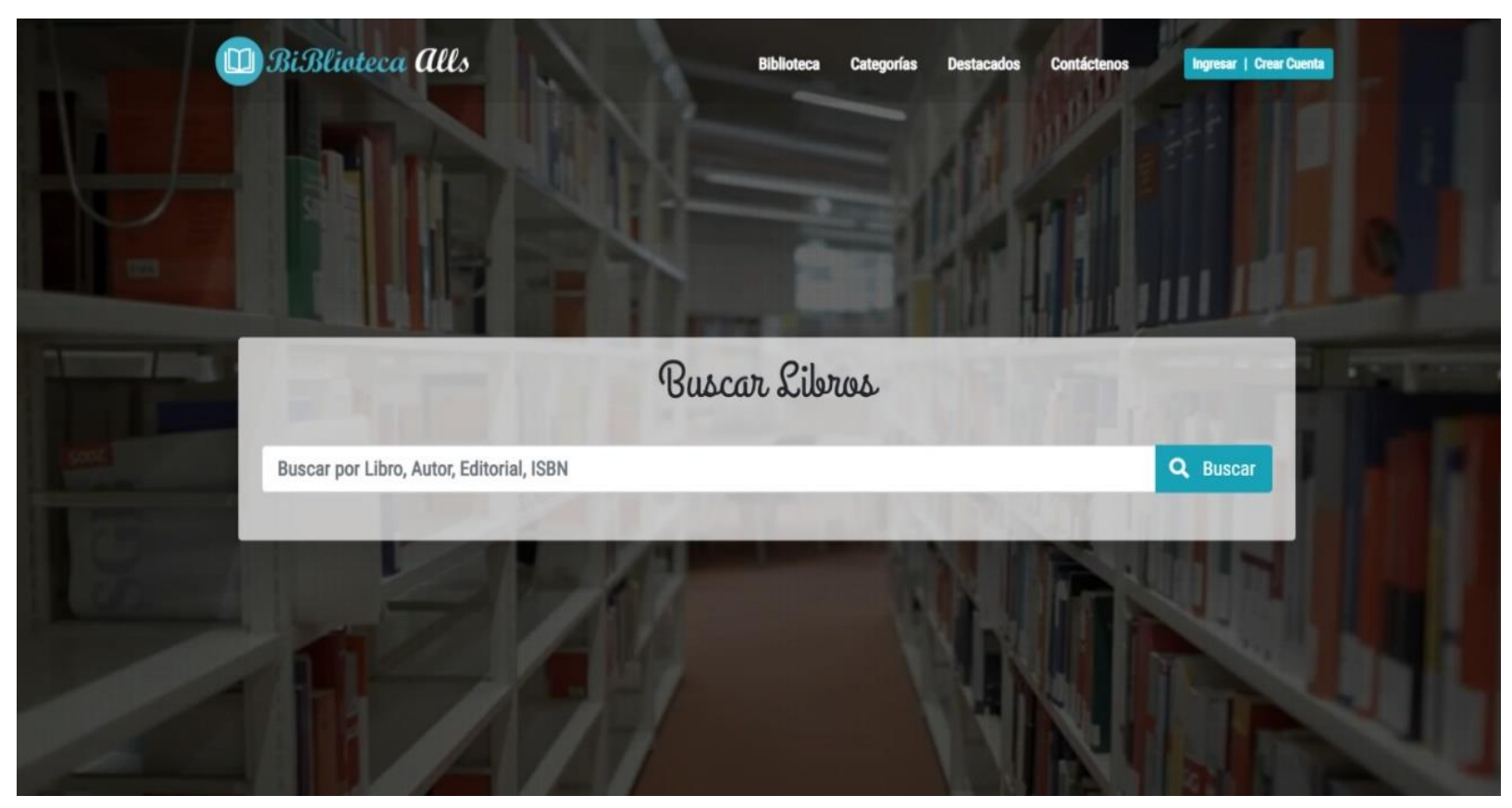

Figura 1. Página principal de la biblioteca virtual.

Fuente: Elaboración propia. 
Revista Arbitrada Interdisciplinaria KOINONIA

Año VI. Vol VI. N¹1. Enero - Junio. 2021

Hecho el depósito de Ley: FA2016000010

ISSN: 2542-3088

FUNDACIÓN KOINONIA (F.K). Santa Ana de Coro. Venezuela.

Axel Alejandro Macías-Rodríguez; Luís Javier Molina-Chalacán; Edmundo Jalón-Arias

\section{Pruebas e implementación}

Para acceder a la aplicación web se debe ingresar a la dirección http://bibliotecaec.online/

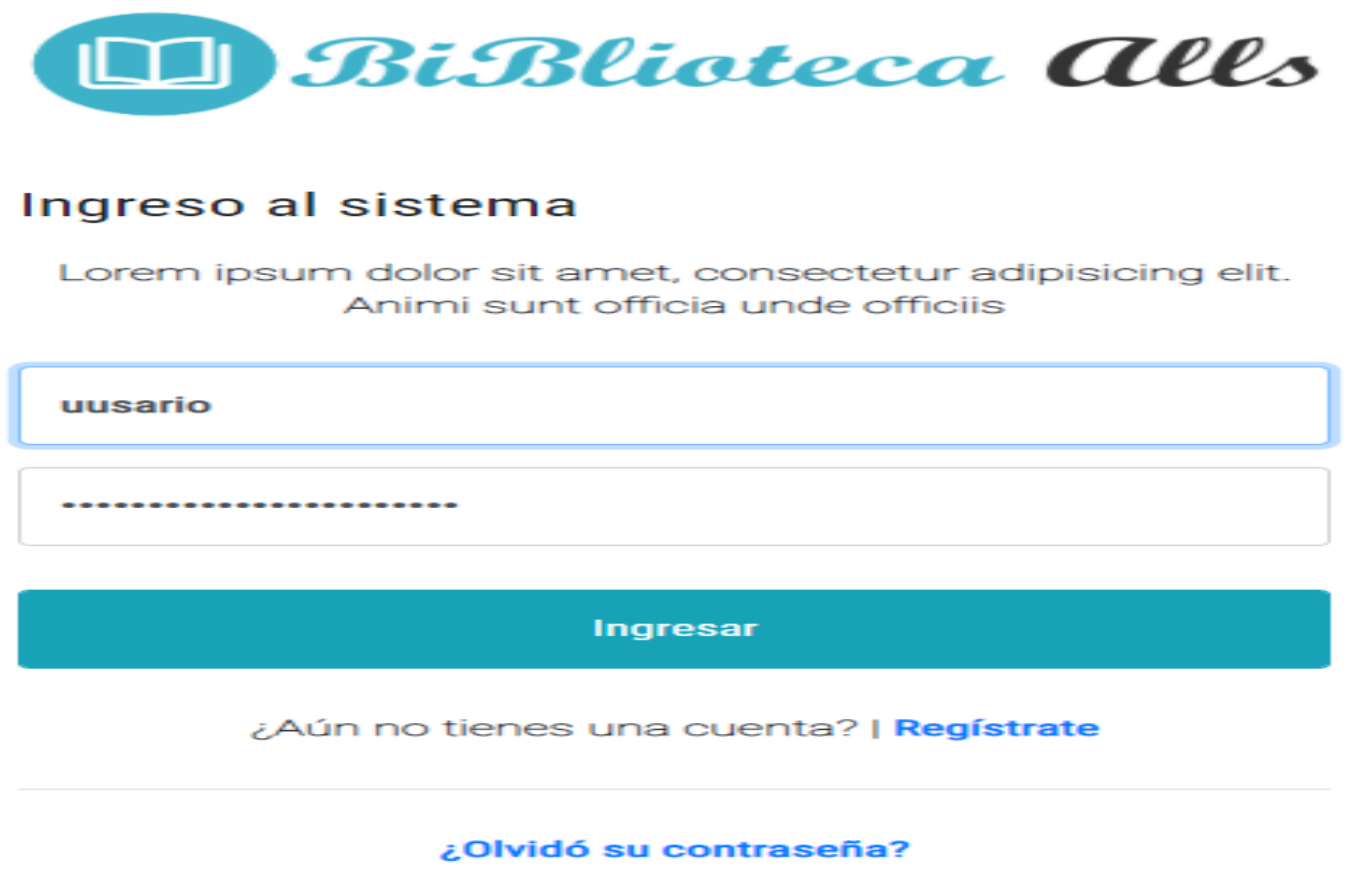

Figura 2. Inicio de sesión de la aplicación web.

Fuente: Elaboración propia. 
Revista Arbitrada Interdisciplinaria KOINONIA

Año VI. Vol VI. N¹1. Enero - Junio. 2021

Hecho el depósito de Ley: FA2016000010

ISSN: 2542-3088

FUNDACIÓN KOINONIA (F.K). Santa Ana de Coro. Venezuela.

Axel Alejandro Macías-Rodríguez; Luís Javier Molina-Chalacán; Edmundo Jalón-Arias

A continuación, se muestra la lista y los reportes de préstamo de un libro en particular:

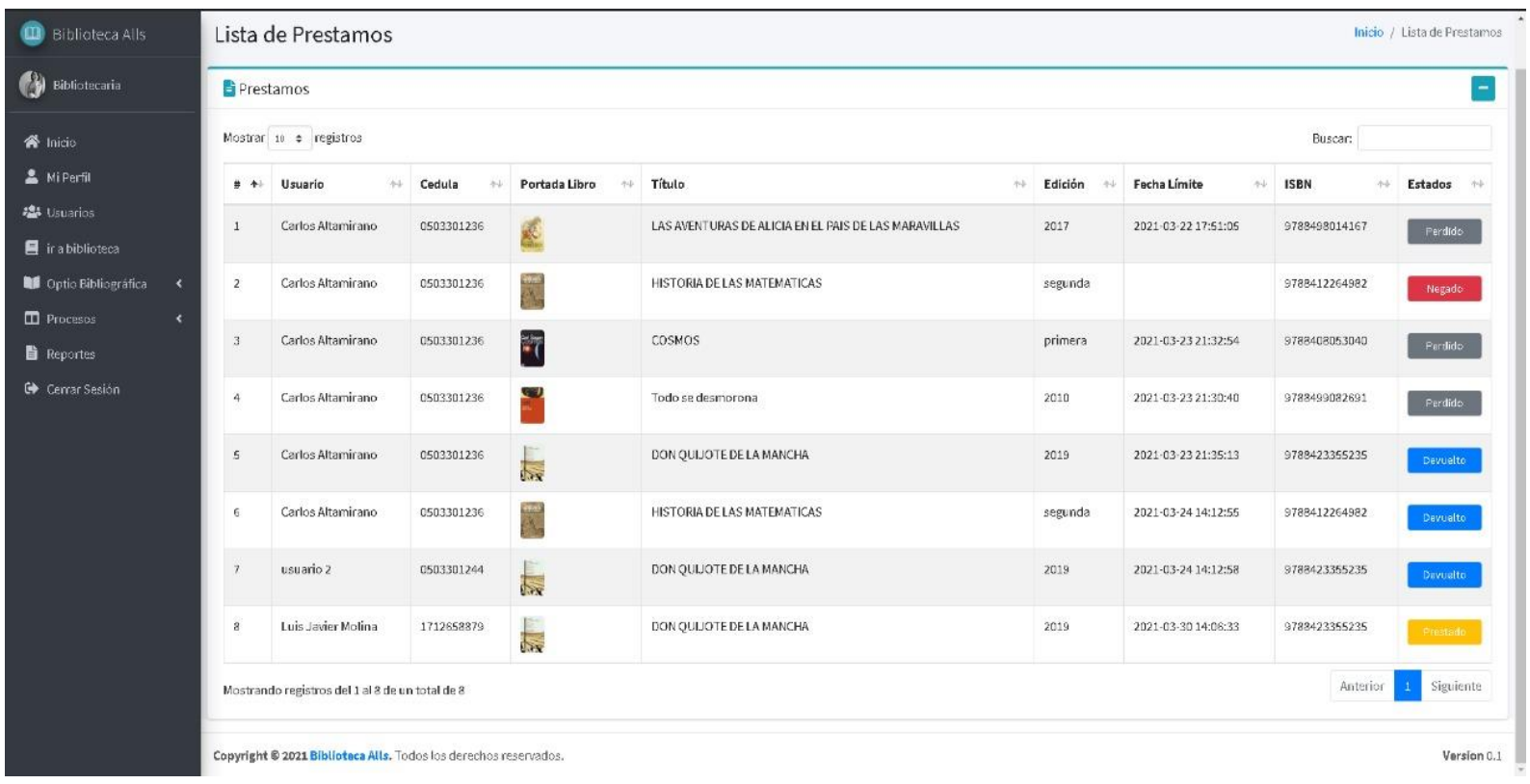

Figura 3. Lista y reportes de préstamo de libros.

Fuente: Elaboración propia.

En la figura 4 se presenta la categoría de los libros

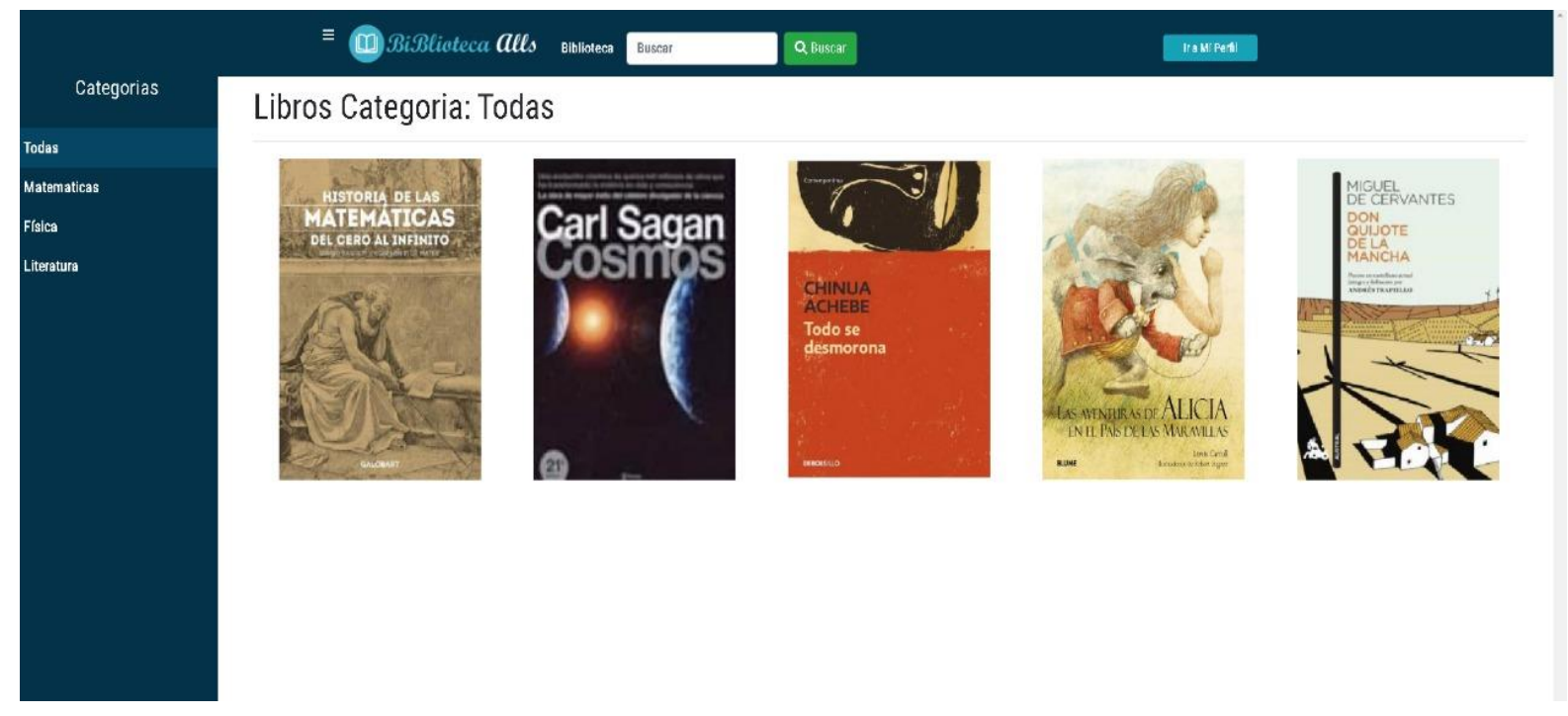

Figura 4. Categoría de libros.

Fuente: Elaboración propia. 
En la figura 5 se muestra la solicitud de un préstamo de libro:

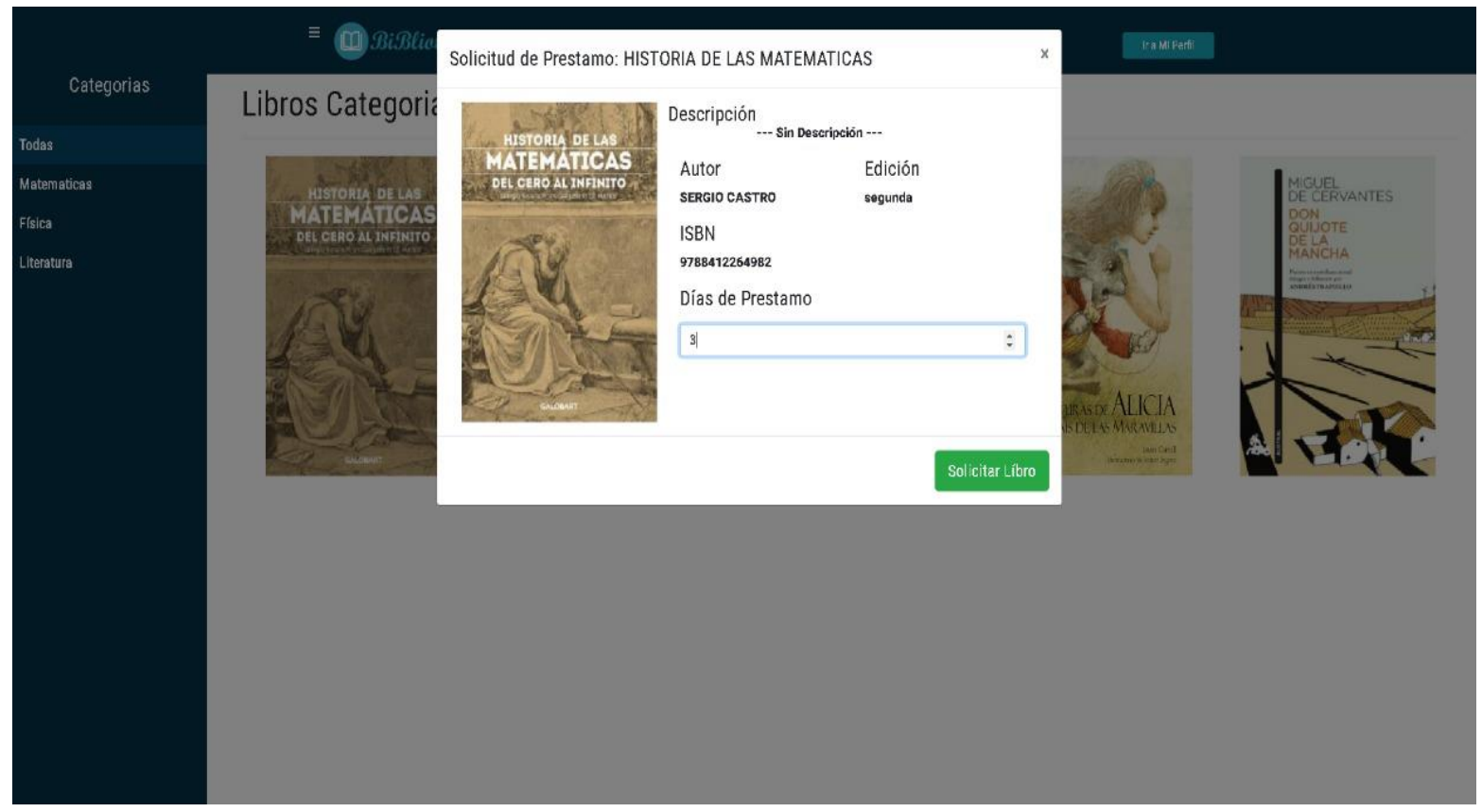

Figura 5. Solicitud de préstamo.

Fuente: Elaboración propia.

\section{CONCLUSIONES}

Se desarrolló la aplicación web para la búsqueda y ubicación de libros donde se evidenció que posee una interfaz más amigable y llamativa, un flujo de código y estructura más eficiente, y reportes con mayor comprensión.

Las pruebas implementadas de implementar el aplicativo web, indican que su funcionamiento es eficiente y adecuado, además permite una óptima búsqueda de los libros que el usuario así lo requiera o necesite, de tal forma que ayuda a ahorrar el factor tiempo. Otro punto importante es que el aplicativo web tiene múltiples beneficios donde el usuario puede registrarse de forma gratuita (sin costo alguno), además cuenta con una 
gran variedad de libros donde pueden solicitarlo a través del admin o el bibliotecario encargado.

Si se emplea una aplicación web para la búsqueda y ubicación de libros, textos, documentos, archivos, entre otros. Son una buena opción para las Instituciones educativas que aún no poseen este tipo de bibliotecas virtuales, ya que es un método más rápido y efectivo para la búsqueda de información que el usuario o estudiante así lo requiera.

La implementación de bibliotecas virtuales en las Instituciones Educativas dentro del país cada día va en aumento, por cuanto no solo las Universidades la poseen sino también estas se encuentran en Instituciones de educación secundaria e incluso de primaria. La importancia de estas bibliotecas tecnológicas es que permite al usuario y/o estudiantes acceder de manera directo a los diferentes puntos de interés, además el acceso en línea permite interactuar con las fuentes de información por medio de la conexión a páginas web.

\section{FINANCIAMIENTO}

No monetario.

\section{AGRADECIMIENTO}

A la Universidad Regional Autónoma de los Andes, Quevedo; por motivar el desarrollo de la investigación.

\section{REFERENCIAS CONSULTADAS}

Aldana-Zavala, J. J. (2017). La Investigación Social: Competencias en el Docente Universitario [Social Research: Competences in the University Teacher]. Fondo Editorial Fundación Koinonía, 3(1), 117. https://doi.org/10.35381/978-980-7792$\underline{13-4}$ 
Carrizo, D, \& Alfaro, A. (2018). Método de aseguramiento de la calidad en una metodología de desarrollo de software: un enfoque práctico [Quality assurance method in a software development methodology: a practice approach]. Ingeniare. Revista chilena de ingeniería, 26(1), 114-129. https://dx.doi.org/10.4067/S0718$\underline{33052018000100114}$

Chiroles-Cantera, M, Pacheco-Correa, Y, Reyes-Chirino, R, \& Sisto-Díaz, A. (2017). Implementación de una aplicación web para el módulo servicio quirúrgico de la aplicación Behique [Implementation of a Web application for the module of surgical service through BEHIQUE system]. Revista de Ciencias Médicas de Pinar del Río, 21(6), 64-71.

Cruz, J. (2011). Herramientas de la web 2.0 aplicadas a la biblioteca virtual UCV caso: Facebook [Web 2.0 tools applied to the UCV virtual library case: Facebook]. Trabajo de titulación. Escuela de Bibliotecología y Archivología. Universidad Central de Venezuela. Disponible en http://eprints.rclis.org/23921/1/tesis.pdf

Guerrero, F, Francisco-Díaz, J, \& Delgado, C. (2015). Aplicación web basada en programación por restricciones para ingeniería de asignación de espectro [A Constraint programming based web application for spectrum allocation engineering]. Ingeniare. Revista chilena de ingeniería, 23(4), 526539. https://dx.doi.org/10.4067/S0718-33052015000400005

Hernández-Pacheco, F. (2013). Investigación y bibliotecas públicas [Research and public libraries]. Investigación bibliotecológica, 27(61), 7-12.

Meneses-Tello, F. (2013). Bibliotecas y sociedad: el paradigma social de la biblioteca pública [Libraries and society: the social paradigm of the public library]. Investigación bibliotecológica, 27(61), 157-173.

Quezada-Sarmiento, P. (2017). Implementación de una solución web y móvil para la gestión vehicular basada en Arquitectura de Aspectos y metodologías ágiles: Un enfoque educativo de la teoría a la práctica [Implementation of a web and mobile solution for vehicular management based on Aspects Architecture and Agile Methodologies: An educational approach from theory to practice]. RISTI - Revista Ibérica de Sistemas e Tecnologias de Informação, (25), 98111. https://dx.doi.org/10.17013/risti.25.98-111 
Sanz, M. (2016). Programación web en el entorno cliente [Web programming in the client environment]. Madrid-España: Editorial RA-MA.

Silva-Blancas, V.H., \& Romero-González, R.M. (2018). Metodología para la obtención de modelos de datos a partir de variables cognitivas [Methodology for retrieving data model as from cognitive variables]. RIIIT. Revista internacional de investigación e innovación tecnológica, 6(35). 